Digital Press Social Sciences and Humanities

L'interférence des langues étrangères dans des méthodes d'enseignement de français : étude sociopragmatique

Nurul Hikmayaty Saefullah, Rosaria Mita Amalia and Savitri Aditiany

Proceeding of Conférence internationale sur le français 2018

Joesana Tjahjani, Merry Andriani, Sajarwa, Wening Udasmoro (eds) 


\title{
L'interférence des langues étrangères dans des méthodes d'enseignement de français : étude sociopragmatique
}

\author{
Nurul Hikmayaty Saefullah*, Rosaria Mita Amalia et Savitri Aditiany \\ Universitas Padjadjaran, Bandung, Indonesia \\ *e-mail: nurul.h.saefullah@unpad.ac.id
}

\section{Résumé}

Le français est le plus fort concurrent de la langue anglaise. L'existence de l'anglais est considérée comme une menace pour la langue française avec l'inclusion d'un grand vocabulaire anglais en français. Les menaces viennent non seulement de l'anglais, mais aussi d'autres langues étrangères, y compris les langues des pays d'immigration vivant en France et la langue des pays voisins de la France. L'interaction de la langue française avec ces langues semble compréhensible et acceptée dans la politique française elle-même. Les méthodes d'enseignement de français comprennent aujourd'hui de nombreux vocabulaires en langues étrangères, qui devraient être libres de toute influence étrangère. Cela montre que le français a commencé à s'ouvrir, que l'apprentissage de la langue devrait également apprendre sur la culture et toutes les choses qui s'y rapportent. Le cas est maintenant, non pas comment l'arrêter, mais comment le contrôler et le voir comme une tentative positive dans l'interaction humaine. C'est le but de cette étude. Linguistiquement, il est intéressant d'étudier cette problématique en utilisant la théorie sociopragmatique, c'est-à-dire en considérant la pratique de l'usage des langues étrangères dans le manuel de la française à partir de la culture et du pragmatisme. En utilisant les données des méthodes d'enseignement de français, l'analyse dans cette étude a été menée en utilisant une méthode analytique descriptive. Les théories utilisées sont des théories sociolinguistiques et pragmatiques. Le résultat attendu de cette étude est la découverte des positifs de l'interférence des langues étrangères dans les méthodes d'enseignement de français et cela ne nuit pas à la réputation du français aux yeux de la communauté internationale.

\section{Mots-clés}

enseignement de français, interférence, sociopragmatique

\begin{abstract}
French is the strongest competitor of the English language. The existence of English is considered to be a threat to French language with the inclusion of a large English vocabulary in French. The threats come not only from English, but also from other foreign languages, including the languages of the countries of immigration living in France and the language of the neighboring countries. The interaction of French with these languages seems understandable and accepted in French politics itself. French teaching methods include today of many vocabularies in foreign languages, which should be free of any foreign influence. It shows that French began to open up, that learning the language should also learn about its culture and all things related. The case is now, not how to stop it, but how to control it and see it as a positive attempt in human interaction. It is the purpose of this study. Linguistically, it is interesting to study this problem using sociopragmatic theory, i.e. considering the practice of the use of foreign languages in the manual of French from the culture and pragmatism. Using data from French teaching methods, the analysis was conducted using a descriptive analytical method, with sociolinguistic and pragmatic as theoretical background. The expected outcome of this study is the discovery of the positives of the interference of foreign languages in the teaching of French methods and it does not harm the reputation of French in the eyes of the international community.
\end{abstract}

\section{Keywords}

French teaching, interference, sociopragmatics 


\section{Introduction}

L'acquisition d'une langue étrangère pourrait être réalisée grâce à plusieurs raisons. L'une de ces raisons est un contact. RebekahRast, une professeure et chercheuse de l'American University of Paris a fait une recherche sur le phénomène de l'acquisition d'une nouvelle langue étrangère chez les francophones qui savent parler au moins deux langues et n'ont jamais eu de connaissance sur le polonais, dans ce cas il est pris comme la troisième langue (L3). Elle a fait cette recherche pour voir la compréhension d'une langue inconnue considérée comme la L3 chez les francophones depuis leur premier contact avec cette langue inconnue.

Au début de l'article, l'auteur parle du concept du transfert de la langue source à la langue cible de Kellerman (1983), dit la psychotypologie. Ce concept explique que le transfert se passe par l'évaluation subjective de l'apprenant pour comprendre la distance typologique entre les langues. Dans ce cas, l'apprenant lui-même qui évalue la distance et la proximité de langue(s) source(s) et langue cible. En prenant ce concept, l'auteur met l'objectif de ses études pour identifier les traits de la première langue (L1), des langues secondes (L2) et de la nouvelle langue cible comme la troisième langue (L3) par un test de traduction de mots.

\subsection{La psychotypologie}

Nous pouvons trouver sur la page lefigaro.fr, Gaël (2017) mentionne qu'il y a 274 millions de personnes autour du monde qui parlent français. Le français est devenu la deuxième langue au monde à être appris après l'anglais. Cela montre que le français n'est pas seulement utilisé dans les pays francophones mais aussi étudié dans des pays hors du francophone, comme l'Indonésie. Selon la Fondation Alliance Française (2018) :

«L'Alliance Française enseigne le Français Langue Étrangère dans plus de 700 centres dans 132 pays. Les Alliances Françaises accueillent chaque année un large public avec plus de 450000 personnes qui viennent apprendre le français. Enfants, adolescents, adultes, étudiants, travailleurs, retraités [...].»

À partir de cette information, nous savons que les apprenants de langue française proviennent de différents âges et professions. Le français est enseigné dans les écoles, les universités, les établissements d'enseignement, ainsi que le cours privé pour les personnes intéressées. En examinant le niveau de formalité de l'enseignement et de l'éducation en français, il faut des enseignants et de bons livres. Comment une méthode de français peut-elle être considéré bonne ? Bien sûr, il existe plusieurs maisons d'édition qui peuvent être considérés comme étant de bonne qualité et conformes aux standards du Cadre Européen Commun de Référence pour les Langues (CECRL), parmi lesquels nous utilisons comme sources de données dans cet article.

Parmi les cinq méthodes de français de niveau débutant que nous avons utilisés comme sources de données, il semble que l'apprentissage de la langue française ne puisse être séparé des interactions avec d'autres langues étrangères, y compris de l'anglais. Ceci est intéressant à étudier, car selon notre hypothèse, l'apprentissage des langues étrangères doit être libre de " contamination » d'autres langues étrangères. Cela peut causer de la confusion chez les apprenants débutants. De telles interactions interlangues indiquent l'existence d'un emprunt du langage dans les méthodes d'apprentissage du français. Deux points importants peuvent être soulevés pour aborder cette question. Premièrement, en termes de linguistique, quelles langues étrangères sont-elles empruntées dans les méthodes d'apprentissage de français et à quel domaine appartient-elles ? Deuxièmement, en termes d'interculturalité, comment cet emprunt peut-il être prouvée d'apporter des effets positifs qui se produit dans l'apprentissage de français?

Les deux points de problématique ci-dessus confirment le but de cette étude, (1) à identifier les langues étrangères empruntées dans les méthodes d'apprentissage du français et ses domaines, et (2) à prouver les effets positifs de l'emprunt, du point de vue de la pragmatique de l'interculturalité.

Pour pouvoir répondre aux problèmes et atteindre les objectifs de la recherche, nous utilisons des théories linguistiques, celles liées à la pragmatique de l'interculturalité. Nous faisons une analyse base à la théorie de pragmatique de l'interculturalité de Kecskes \& Horn (2007), et les autres théories liées comme la relation entre langue et culture de Kramsch (1998), la sociolinguistique et l'apprentissage des langues 
de Hornberger \& McKay (2010), l'emprunt de Moreau (ed.) (1997), et le bilinguisme d'Appel \& Muysken (1987) et Grosjean (2008).

\section{Méthodologie de recherche}

La linguistique comme science humaine traite spécifiquement de la langue qui est le moyen de communication. Dans cet article, nous nous concentrons à la pragmatique de l'interculturalité, car elle peut voir l'utilisation de la langue non seulement en termes intralingues, mais aussi extralingues. Mey dans Kecskes \& Horn (2007, p. 172) croit que pour la pragmatique de l'interculturalité, l'utilisation de la langue ne peut être séparée de l'utilisateur et de son contexte social. Le contexte social provient de milieux culturels, à la fois intraculturels et interculturels. Sur la base de ces avis, nous ne pouvons pas nier l'existence d'un lien entre la langue, ses utilisateurs et son contexte culturel. Le lien entre les trois éléments soulève l'identité culturelle. Kramsch (1998, p. 65) déclare qu'il y a un lien naturel entre la langue parlée par les membres d'un groupe social et l'identité de ce groupe à partir de leur accent, leur vocabulaire et les modèles de discours. À l'ère de la mondialisation, l'identité d'une communauté est importante car cette identité distingue une communauté linguistique d'une autre communauté linguistique. En tant qu'êtres sociaux, les membres d'une communauté font une interaction avec les membres d'autres communautés. En bref, c'est ce qu'on appelle l'interculturalité.

Dans l'apprentissage de la langue française, l'interculturalité est très importante pour être discutée dans les méthodes. En plus d'introduire la langue et la culture françaises, les méthodes en français mènent désormais à la socialisation linguistique. Duff (dans Hornberger \& McKay, 2010, pp. 427-452) exprime que l'apprentissage de la langue nécessite une socialisation de la langue qui aide les apprenants à maitriser la langue qu'ils apprennent. Bien entendu, cette socialisation doit s'accompagner d'une compréhension de la culture correspondant au matériel linguistique appris. Ce qu'il faut noter, c'est que lorsque deux langues sont en contact, une langue emprunte des mots d'autres langues. Selon Hamers (dans Moreau, 1997, pp. 136-140), l'emprunt est un mécanisme normal de l'évolution linguistique. Le phénomène de l'emprunt dans les méthodes de français assure que les apprenants de français deviennent des bilingues et même des multilingues (voir Appel \& Muysken, 1987 ; Grosjean, 2008).

Pour bien faire la recherche, nous utilisons cinq méthodes de français pour les débutants universitaires, ce sont : Nouveau Rond-Point 1 (Flumian et al, 2012), Totem 1 (LeBougnec \& Lopes, 2014), Entre Nous 1 (Pruvost et al, 2015), Tendance 1 (Girardet et al, 2016) et Cosmopolite 1 (Hirschsprung \& Tricot, 2017). Les données sont prises en observant ces méthodes d'apprentissage. Ensuite, nous vérifions les données en utilisant le dictionnaire, le but est pour assurer les mots étrangers du point de vue de leur sens et de leur étymologie. L'analyse de données est commencée par classifier les données selon leur domaine (alphabétiquement). Après avoir faire les classifications, nous faisons une analyse plus approfondie pour répondre aux problématiques questionnées. Pour analyser les données, nous appliquons la méthode de l'analyse descriptive pour que les résultats restent objectifs.

Nous collectons 242 donées de cinq méthodes d'apprentissage. Les données sont divisées en classifications des domaines de la vie. Voici l'explication de chaque classification :

(1) L'activité ; dans cette classification, nous trouvons quatorze données, entre outre brunch (de l'anglais), feng shui (du chinois) et sieste (de l'espagnol). L'anglais est la langue dominante dans cette classification $(78,57 \%)$.

(2) La communication ; Les données dans cette classification par exemple buzz média, couchsurfer et speakdating. Toutes les sept données qui appartient au domaine de la communication sont de l'anglais.

(3) Les danses ; Flamenco, rumba, salsa (de l'espagnol) et samba (du portugais) sont les données obtenues du domaine des danses.

(4) L'état ; Dans ce domaine, nous essayons de vous montrer les mots liés à notre état physique ou bien psychologique, comme cool, hype, superwoman (de l'anglais) et zen (du japonais). 83,3\% des données sont de l'anglais.

(5) Les événements culturels ; Il y a des mots-valises au domaine culturel trouvés dans les méthodes d'apprentissage de français, par exemple coolture, hellfest et solidays (de l'anglais). Il n'y a qu'un mot arabe apparait dans ce domaine (Aïd).

(6) Les jeux; Nous ne trouvons que cinq données dans cette clasification et quatre d'entre elles sont de l'anglais : joker, laser game, playmobil et rallye. La cinquième donnée vient de l'arabe : baraka.

(7) Les lieux; L'anglais est encore la langue dominante dans ce domaine, $80 \%$ de données sont de l'anglais, par exemple camping, cooking school, speaker's corner, même le nom de pays des ÉtatsUnis est écrit en anglais : USA. Les autres mots sont de l'hindi, bungalow et du portugais, pagode. 
(8) Les modes ; Les données sont de l'anglais $(87,5 \%)$ et de l'hindi, par exemple dressing, modesign, short et sari.

(9) La musique : les genres de musique comme blues passions, country et hardrock (de l'anglais) sont une petite partie des données dans cette classification. Il y a treize données au domaine de la musique sont de l'anglais. Les autres données mentionnées dans les méthodes sont djembé (une sorte d'instrument de musique africain), zouk (de l'arabe), reggae sun ska festival (estimé venir d'un dialecte africain) et karaoké (du japonais).

(10) Les noms propres ; Cette classification est la plus nombreuse (66 données) et très variées. Les noms cités par exemple Mathias (de l'allemand), John (de l'anglais), Karim (de l'arabe), Chang (du chinois), Ji Woong (du coréen), Lopez (de l'espagnol), Satya (de l'hindi), Luigi (de l'italien), Aiko (du japonais), Agneska (du polonais), Felipe (du portugais), Vladimir (de la russe), Chao Fah (du thailandais) et Thu Anh (du vietnamien). Le pourcentage des langues dans cette classification est presque équilibre.

(11) Les objets ; Il n'y a que trois données dans cette classification, et elles viennent de l'anglais : kit de survie, patchwork et ring.

(12) Les professions ; La langue dominante dans cette classification est l'anglais (91,67\%). Les mots apparus par exemple baby-sitter, cameraman, leader et salarymen. Un mot japonais complète la liste, sumotori.

(13) Les salutations ; La liste est très variée bien que les données ne soient pas très nombreuses (huit données). Nous pouvons voir les mots hallo (de l'allemand), hello (de l'anglais), ni hao (du chinois), hola (de l'espagnol), ciao (de l'italien), tak.słucham (du polonais) et diga (du portugais).

(14) Les sports ; Ce domaine emprunt beaucoup de l'anglais (80,95\%). Les 21 données apparues par exemple canyoning, handball, snowboard, stretching (de l'anglais), kung-fu (du chinois), karaté (du japonais) et yoga (du sanskrit).

(15) Les technologies de l'information ; La liste de cette classification est remplie de l'anglais (10 données), comme antispam, cookening, start-up, tweeet, etc.

(16) Les termes culinaires; Le domaine des termes culinaires vient au deuxième rang après les noms propres. Il est aussi très varié. Nous pouvons trouver les mots comme cookies, hamburger, fast-foods (de l'anglais), couscous (de l'arabe), guacamole (de l'espagnol), lassi (de l'hindi), cannelloni (de l'italien), sushi (du japonais), speculoos (du néerlandais), feijoada (du portugais), blini (de la russe), banh mi (du vietnamien) et brocciu (du dialecte de Corse) dans la liste de données.

(17) Les transports ; Nous ne découvrons que les mots tramway (de l'anglais) et tuk-tuk (du thaïlandais) dans cette classification.

(18) Les autres domaines ; Cette classification est spécialement conçue pour collecter des données qui ne sont pas incluses dans la classification mentionnée précédemment, par exemple toubab (un dialecte africain), blizzard, coworking, made in France (de l'anglais), etc.

L'anglais est la langue très populaire à emprunter car il est la langue du globe et il fait beaucoup d'influence presque dans tous les domaines de la vie. Il est inséré dans les sources de données pour 132 mots. Quelques d'entre eux sont déjà apparus dans le dictionnaire de français, le reste est de nouveaux mots. Les mots connus appartiennent aux domaines des modes, des genres de musique, des noms propres, des termes culinaires et des sports. Ce sont les domaines dont les pays anglophones sont plus dominants bien que ce résultat soit discutable. Le seul domaine où l'anglais est exclu c'est la danse. L'espagnol et l'italien sont les langues suivantes l'anglais à emprunter. Nous sommes surs que c'est à cause de la géographie. L'Espagne et l'Italie sont les pays voisins de la France et il est raisonable de les emprunter et les présenter dans les méthodes d'apprentissage de français. En plus, le chiffre d'immigration de ces deux pays est élevé (Centre d'observation de la société, 2018). L'arabe, avec 12 mots, vient après. Nous connaissons qu'en France les immigrés des pays d'Afrique notamment le Maghreb dont l'arabe est la langue parlée sont très nombreux. Nous croyons que c'est la raison principale pourquoi l'arabe est emprunté.

Un fait éttonant est indiqué par les langues des pays d'Asie. Nous savons qu'il y a quelques pays d'Asie qui parle français, par exemple le Cambodge, le Laos et le Vietnam. Dans les cinq méthodes que nous utilisons pour recuperer les données, nous trouvons que le japonais et le chinois sont riches, autre que le thaïlandais, le vietnamien et le coréen. Les emprunts sont du domaine des activités, d'état, des musiques, des noms propres, des nourritures, des professions, des salutations et des sports. L'hindi est aussi montré dans les méthodes. Les autres langues empruntées sont des langues d'autres pays d'Europe, par exemple l'allemand, le portugais, le polonais, le néerlandais et le russe. Ces langues ne paraissent pas trop nombreuses dans les sources. 


\section{Discussion}

Les langues étrangères empruntées viennent de douze langues différentes, ce sont : (1) l'anglais ; (2) l'espagnol ; (3) l'italien ; (4) l'arabe ; (5) le japonais ; (6) l'allemand ; (7) le chinois ; (8) le hindi (y compris le sanskrit) ; (9) le portugais ; (10) les langues des pays d'Afrique (y compris les dialectes sénégalais, marocain et jamaican) ; (11) les langue des pays Asie (y compris le thaïlandais, le vietnamien et le coréen) ; et (12) les langues d'autres pays d'Europe (y compris le polonais, le néerlandais, le russe et le dialecte de Corse). Les emprunts appartiennent aux dix-huit domaines, ce sont : (1) les activités ; (2) la communication ; (3) les danses ; (4) l'état ; (5) les événements culturels ; (6) les jeux ; (7) les lieux ; (8) les modes ; (9) la musique ; (10) les noms propres ; (11) les objets ; (12) les professions ; (13) les salutations ; (14) les sports ; (15) les technologies de l'information ; (16) les termes culinaires ; (17) les transports ; et (18) les autres domaines.

À notre avis, les mots empruntés dans les méthodes d'apprentissage de français sont des mots très proches de la culture française ou des mots que l'on peut facilement trouver dans l'interaction humaine. Nous voyons que les mots inclus dans les domaines de la musique, des termes culinaires, des sports et des modes sont les domaines de la vie très familiers avec les langues étrangères, en particulier avec l'anglais. Ces termes sont étroitement liés à la culture française bien connue dans ces domaines. Les autres domaines sont des domaines qui sont peu à peu empruntés, non pas parce que le français n'a pas le bon équivalent pour ces mots, mais on ne peut nier que les termes empruntés sont plus faciles à dire dans la langue d'origine, par exemple les domaines des activités, de la communication, de l'état physique, des evenements culturels, des technologies de l'information et des transports. Les mots empruntés proviennent principalement de l'anglais qui est dominant dans le monde, outre que les mots des langues des pays voisins, des immigrés et des touristes qui viennent en France. L'interaction entre le français et les langues étrangères dans les méthodes d'apprentissage de français est inévitable. Comme nous le savons, l'apprentissage de la langue doit s'accompagner de l'apprentissage de la culture. Ainsi, lorsque nous voulons bien connaître le français, nous devons également présenter la culture française qui ne peut être séparée de sa socialisation avec d'autres cultures très proches en France. Non seulement la culture française est introduite dans les méthodes, lorsque la langue étrangère est empruntée, sa culture est aussi présentée. Ici, nous pouvons voir l'effet positif, les apprenants sont invités à voyager dans le monde entier pour connaître d'autres cultures que la culture française. Bien sûr, il y a aussi des effets négatifs, lorsque trop de langues étrangères apparaissent dans les méthodes, il est possible que les apprenants remplacent facilement les termes français qu'ils ne connaissent pas avec la langue de leur culture ou d'autres cultures étrangères. Donc, il y a des côtés positifs et négatifs de l'emprunt, À partir des résultats de la recherche, les effets positifs et négatifs de l'emprunt restent toujours intéressants à discuter.

\section{Conclusion}

La langue ne peut pas être stérile de l'interaction avec d'autres langues qui l'entourent. Par conséquent, ce serait bien si nous empruntions des termes étrangers, nous puissions faire adopting and adapting, les emprunter mais aussi les adapter à notre propre langue, à notre propre culture. Cela s'applique également pour l'apprentissage du français.

\section{Références}

Appel, R., \& Muysken, P. (1987). Language Contact and Bilingualism. Amsterdam: University of Amsterdam.

Centre d'observation de la société. (2018). D’où viennent les immigrés ? Retrieved from

http://www.observationsociete.fr/population/immigres-et-etrangers/dou-viennent-lesimmigres.html

Duff, P. A. (2010). Language Socialization. In N. Hornberger \& S. L. McKay (Eds.), Sociolinguistics and Language Education (New Perspectives on Language and Education) (p. 427). Bristol: Multilingual

Matters. 
Flumian, C., Labascoule, J., Lause, C., \& Royer, C. (2012). Nouveau Rond-Point 1 : Méthode de français basée sur l'apprentissage par les tâches. Paris: Éditions Maison des Langues.

Fondation Alliance Française. (2018). Apprendre le français comme langue étrangère (FLE). Retrieved from https://www.fondation-alliancefr.org/?page_id=28412

Girardet, J., Pécheur, J., Gibbe, C., \& Parizet, M.-L. (2016). Tendance : Méthode de français A1. Paris : CLE International. Paris: CLE International.

Grosjean, F. (2008). Studying Bilinguals. Oxford: Oxford University Press.

Hamers, J. F. (1997). Emprunt. In Sociolinguistique : Concept de base (2nd ed., pp. 136-140). Liege: Editions Mardaga.

Hirschsprung, N., \& Tricot, T. (2017). Cosmopolite 1 : Méthode de français A1. Paris: Hachette Français Langue Étrangère.

Kramsch, C. (1998). Language and Culture. Oxford: Oxford University Press.

LeBougnec, J.-T., \& Lopes, M.-J. (2014). Totem 1 : Méthode de français A1. Paris : Hachette Français Langue Étrangère. Paris: Hachette.

Mey, J. L. (2007). Developping Pragmatics Interculturally. In I. Kecskes \& L. R. Horn (Eds.), Explorations in Pragmatics : Linguistics, Cognitive and Intercultural Aspects (pp. 165-189). Berlin: Mouton de Gruyter.

Pruvost;, N., Courteaud, F., Gómez-Jordana, Sonia; Puren, C., \& Agustín, G. (2015). Pruvost, N., Courteaud, F., Gómez-Jordana, S., Blondel, F., Chahi, F., Caballero, G., ...Brandel, K. (2015). Entre Nous 1 : Méthode de français. Paris: Éditions Maison de Langues. 